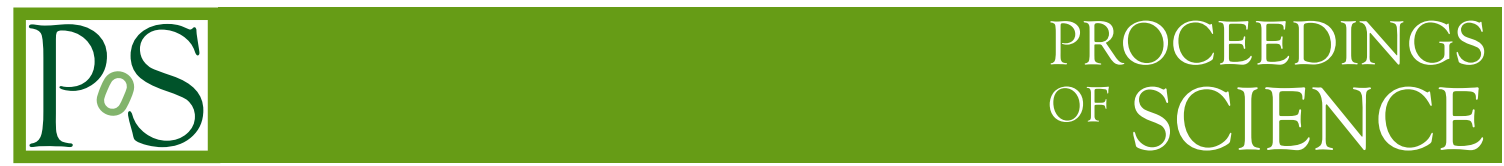

\title{
Exotics searches in photon and lepton final states with the ATLAS detector
}

\section{Tetiana BERGER-HRYNOVA*† \\ $L A P P$ \\ E-mail: thrynova@mail.cern.ch}

This paper presents a summary of exotics searches with photons and leptons in the final state performed up to July 2011 with the proton-proton collision data collected at the LHC at the center-of-mass energy of $7 \mathrm{TeV}$ in 2010 and 2011 by the ATLAS Collaboration.

XXIst International Europhysics Conference on High Energy Physics

21-27 July 2011

Grenoble, Rhônes-Alpes, France

*Speaker.

$\dagger$ on behalf of the ATLAS Collaboration 


\section{Introduction}

The Standard Model (SM) of particle physics describes quite well the data obtained from highenergy experiments to date, but it is thought to be only a low-energy effective theory. Arguments based on unitarity require new physics at the $\mathrm{TeV}$ scale. With $7 \mathrm{TeV}$ centre-of-mass energy protonproton collisions, the LHC is able to probe directly physics at such scales. At this energy the total cross section is about ten orders of magnitude higher than any signal expected from new physics. Photons and charged leptons (electrons and muons) in the final state, described in this paper, enable an effective background suppression and thus provide distinctive signatures for such new processes. This paper will cover a wide range of subjects such as searches for new heavy gauge bosons, compositeness, extra dimensions, lepto-quarks and highly-ionizing particles. For discussion of the other non-lepton(photon) final states see Ref. [1].

ATLAS [2] is a general purpose detector at the LHC. Systems relevant for the photon and electron identification are the inner-detector system, immersed in a $2 \mathrm{~T}$ axial magnetic field with charged particle tracking in the range $|\eta|<2.5$, and a sampling Liquid Argon electromagnetic calorimeter with coverage $|\eta|<4$.9. Systems relevant for the muon reconstruction are the inner detector and the muon spectrometer, which is an instrumented air-toroid magnetic system with precision chamber coverage $|\eta|<2.7$.

Up to July 2011, ATLAS detector has recorded $45 \mathrm{pb}^{-1}$ of proton-proton collision data in the year 2010 and $1.23 \mathrm{fb}^{-1}$ in 2011 . The latter data has on average $\approx 6$ interactions per bunch crossing. The analyses presented in this paper have used various subsets of the data-sets above.

\section{Di-lepton searches}

One of the most popular final states to search for new physics is di-leptons.

It was used in searches for narrow heavy gauge boson resonances, such as Sequential SM $Z^{\prime}$, and Randall-Sundrum (RS) Gravitons. No excess over the SM expectations was seen in the dilepton mass spectrum in $\approx 1 \mathrm{fb}^{-1}$ of 2011 data [3]. Combining di-electron and di-muon channels, $95 \%$ confidence level upper limits on the resonance masses were set at $1.83 \mathrm{TeV}$ for Sequential SM $Z^{\prime}$ and $1.63 \mathrm{TeV}$ for RS Graviton at coupling $k / M_{P l}=0.1$. This is the first time those limits exceed both TeVatron direct limits and indirect limits from the LEP experiments.

Also a search for a non-resonant excess in this channel was performed with $42 \mathrm{pb}^{-1}$ of 2010 data [4]. No excess over the SM expectations was seen in the di-muon mass spectrum and using a left-left isoscalar model a limit was set on the quark-lepton compositeness energy scale $(\Lambda)$ at 4.9(4.5) TeV assuming constructive(destructive) interference between the SM Drell-Yan production and the new physics processes.

There was also another search performed in the same-sign muon channel with $31 \mathrm{pb}^{-1}$ of 2010 data [5]. No excess over the SM expectation was seen in the track multiplicity distribution. A model-independent upper limit at $95 \%$ confidence level of $0.184 \mathrm{pb}$ on the cross section times acceptance times branching fraction was set in this channel and more detailed interpretations in a low scale gravity model were given. 


\section{Di-photon searches}

A search for the RS Graviton in the di-photon channel was performed with $31 \mathrm{pb}^{-1}$ of 2010 data [6]. No excess over the SM expectations was seen and an upper limit at $95 \%$ confidence level on the mass of RS Graviton was set at $0.92 \mathrm{TeV}$ for coupling $k / M_{P l}=0.1$. As the RS Graviton to di-photon decay branching fraction is two times higher than that in the di-lepton channel, major improvements in this measurement can be expected when the analysis is updated to higher luminosity.

\section{Di-photon with missing transverse energy search}

Some new physics signatures result in an excess of missing transverse energy. A study of missing transverse energy above $125 \mathrm{GeV}$ was performed in the di-photon channel with $36 \mathrm{pb}^{-1}$ of 2010 data and no excess over the expected SM backgrounds was seen [7]. In the context of a specific model of one $\mathrm{TeV}^{-1}$-size universal extra dimension, an upper limit at $95 \%$ confidence level of $0.18-0.23 \mathrm{pb}$ on the cross section was set. Also a lower limit of $1 / R>961 \mathrm{GeV}$ was set on the universal extra dimension compactification radius $R$.

\section{Lepton with missing transverse energy search}

A search for an excess of events in the transverse mass of a lepton and the missing transverse energy system [8] was performed. No excess over the SM background expectations was seen in $1.04 \mathrm{fb}^{-1}$ of data. Combining electron and muon channels, a $W^{\prime}$ with Sequential SM couplings was excluded at $95 \%$ confidence level for masses up to $2.15 \mathrm{TeV}$.

\section{Leptons with jets searches}

A search for the pair production of first or second generation scalar lepto-quarks was performed using $35 \mathrm{pb}^{-1}$ of 2010 data [9]. Lepto-quarks were searched in events with two oppositely charged muons or electrons and at least two jets, and in events with one muon or electron, missing transverse energy and at least two jets. After event selection, the observed yields were consistent with the predicted backgrounds. Lepto-quark production was excluded at 95\% confidence level for masses $\mathrm{m}_{L Q}<376(319) \mathrm{GeV}$ and $\mathrm{m}_{L Q}<422(362) \mathrm{GeV}$ for first and second generation scalar leptoquarks, respectively, when assuming the branching fraction of a lepto-quark to a charged lepton to be equal to $1.0(0.5)$.

Also a study of the invariant mass distribution of jet pairs produced in association with a leptonically decaying $\mathrm{W}$ boson was performed with $1.02 \mathrm{fb}^{-1}$ of 2011 data. This channel is particularly interesting because of an excess observed by CDF in the $120-160 \mathrm{GeV}$ di-jet mass range [10]. Combining the data from the electron and the muon channels, the measured di-jet mass spectrum showed no significant excess over the Standard Model expectation for di-jet masses ranging up to $400 \mathrm{GeV}[11]$. 


\section{Search for Highly-Ionizing Particles}

A search was made for massive long-lived highly-ionizing particles using $3.1 \mathrm{pb}^{-1}$ of 2010 data [12]. The signature of energy loss in the inner detector and electromagnetic calorimeter was used. No such particles are found and limits on the production cross section for electric charges $6 e \leq|q| \leq 17 e$ and masses $200 \mathrm{GeV} \leq \mathrm{m} \leq 1000 \mathrm{GeV}$ are set in the range $1-12 \mathrm{pb}$ for different hypotheses on the production mechanism.

\section{Summary}

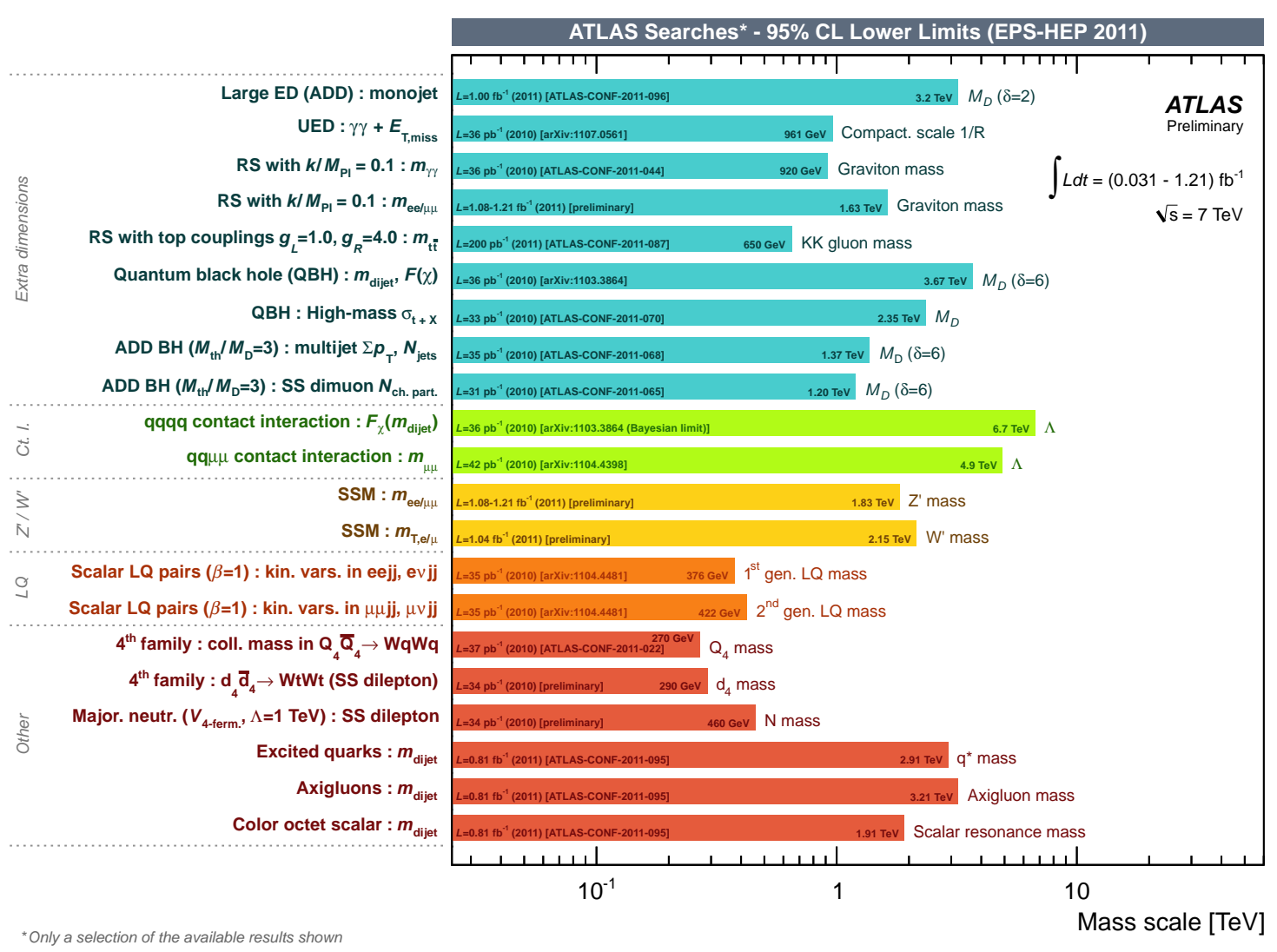

Figure 1: Mass reach of ATLAS searches for new phenomena other than SUSY [13]. Only a representative selection of the available results is shown.

As presented in this paper, the ATLAS Collaboration has so far searched for a wide range of new physics phenomena in channels which include a lepton or a photon in the final state. From Figure 1 one can see that the mass reach for many of these searches is well above $1 \mathrm{TeV}$. No new physics has been seen, but up to the end of 2011 we will have accumulated over four times more data than used for the analysis presented in this paper. Most of those analysis are being updated and the search for new physics is continuing. 


\section{References}

[1] See T. Kuhl and A. Gibson, in these Proceedings.

[2] ATLAS Collaboration, JINST 3, S08003 (2008).

[3] ATLAS Collaboration, arXiv:1108.1582[hep-ex], to be published in Phys. Rev. Lett.

[4] ATLAS Collaboration, Phys. Rev. D 84, 011101(R) (2011).

[5] ATLAS Collaboration, ATLAS-CONF-2011-065, http://cdsweb.cern.ch/record/1346080.

[6] ATLAS Collaboration, ATLAS-CONF-2011-044, http://cdsweb.cern.ch/record/1338573.

[7] ATLAS Collaboration, arxiv:1107.0561[hep-ex], submitted to EPJC Letters.

[8] ATLAS Collaboration, Phys. Lett. B 705, 28 (2011).

[9] ATLAS Collaboration, Phys. Rev. D 83, 112006 (2011).

[10] CDF Collaboration, Phys. Rev. Lett. 106, 171801 (2011).

[11] ATLAS Collaboration, ATLAS-CONF-2011-097, http://cdsweb.cern.ch/record/1369206.

[12] ATLAS Collaboration, Phys. Lett. B 698, 353 (2011).

[13] https://twiki.cern.ch/twiki/bin/view/AtlasPublic/CombinedSummaryPlots?rev=6 